PROCEEDINGS OF THE

AMERICAN MATHEMATICAL SOCIETY

Volume 133, Number 11, Pages 3345-3353

S 0002-9939(05)07893-7

Article electronically published on June 20, 2005

\title{
POISSON KERNELS AND SPARSE WAVELET EXPANSIONS
}

\author{
LORENZO BRANDOLESE
}

(Communicated by David R. Larson)

\begin{abstract}
We give a new characterization of a family of homogeneous Besov spaces by means of atomic decompositions involving poorly localized building blocks. Our main tool is an algorithm for expanding a wavelet into a series of dilated and translated Poisson kernels.
\end{abstract}

\section{INTRODUCTION}

One of the most striking properties of wavelet bases is that functions whose wavelet expansion is sparse (or lacunary) usually have remarkable geometric features. For example, quasi-every sum (in the sense of Baire categories) of a sparse wavelet series is multifractal. This means that the sets where it has a given pointwise Hölder exponent form an infinite collection of fractal sets with different Hausdorff dimension. A crucial fact for the applications is that, in some sense, the converse is also true: many functions arising in signal analysis or in turbulence turn out to have a sparse wavelet expansion, essentially because of their nice geometrical features (see [8], 9]).

A sparse wavelet expansion in $\mathbb{R}^{n}$ is a series of the form

$$
f(x)=\sum_{\psi \in F} \sum_{j \in \mathbb{Z}} \sum_{k \in \mathbb{Z}^{n}} \alpha(j, k) \psi\left(2^{j} x-k\right), \quad x \in \mathbb{R}^{n},
$$

where

$$
\alpha(j, k)=2^{n j} \int f(x) \bar{\psi}\left(2^{j} x-k\right) d x,
$$

$F$ is a finite set of orthogonal wavelets and only a few coefficients $|\alpha(j, k)|$ are larger than fixed small thresholds.

In this paper we mostly deal with infinitely sparse wavelet series which are defined by the condition that the nonincreasing rearrangement $\left(c_{n}^{*}\right)_{n=1}^{\infty}$ of the sequence $|\alpha(j, k)|$ is rapidly decreasing as $n \rightarrow \infty$. This condition can also be expressed by the fact that the wavelet coefficients belong to all the $\ell^{p}\left(\mathbb{Z} \times \mathbb{Z}^{n}\right)$ spaces:

$$
\sum_{j \in \mathbb{Z}} \sum_{k \in \mathbb{Z}^{n}}|\alpha(j, k)|^{p}<\infty \quad \text { for all } p>0 .
$$

Received by the editors March 22, 2004 and, in revised form, June 23, 2004.

2000 Mathematics Subject Classification. Primary 42C40, 41A30.

Key words and phrases. Besov spaces, nonlinear approximation.

(C)2005 American Mathematical Society 
We also know (see 12], 7]) that (1.1)-(1.3) is equivalent to $f \in \bigcap_{p>0} \dot{B}_{p}^{n / p, p}\left(\mathbb{R}^{n}\right)$, where $\dot{B}_{p}^{s, q}\left(\mathbb{R}^{n}\right)(s \in \mathbb{R}, p \geq 0$ and $q \geq 0)$ denotes the homogeneous Besov space (here and below, we assume that the wavelets belong to the Schwartz class).

Recently, there has been an increasing interest in expressing general functions as series of dilated and shifted fixed functions other than wavelets. One important motivation is that this leads to new characterizations of the classical functional spaces. For example, let $G$ be the gaussian and $G_{\lambda, x_{0}}(x)=G\left(\lambda\left(x-x_{0}\right)\right)$, with $\lambda>0$ and $x_{0} \in \mathbb{R}^{n}$ and consider the family of all functions $f$ such that

$$
f(x)=\sum_{j} \alpha_{j} G_{\lambda_{j}, x_{j}}(x) \quad\left(\text { with } \alpha_{j} \in \mathbb{C}, \lambda_{j}>0 \text { and } x_{j} \in \mathbb{R}^{n}\right) .
$$

If $\left(\alpha_{j}\right)$ describes $\ell^{1}$, then the sums of all the series (1.4) exactly give the well-known bump algebra, which has been identified by Meyer [12] to $\dot{B}_{1}^{n, 1}\left(\mathbb{R}^{n}\right)$ and plays an important role in modelling signals with special singularities (see [5]).

We mention also the work of Kyriakis and Petrushev [10, who exhibited a large class of unconditional bases for the Besov and the Triebel-Lizorkin spaces. They proved that such bases can be generated from a small number of shifts and dilates of a single function $\Phi$. For this, they only have to assume that $\Phi$ is smooth enough and that it decays at infinity sufficiently fast. As an application of their result in the case $\Phi=G$, it follows that the topological vector space $\bigcap_{p>0} \dot{B}_{p}^{n / p, p}\left(\mathbb{R}^{n}\right)$ contains exactly the series (1.4), where $\left(\alpha_{j}\right)$ describes $\bigcap_{p>0} \ell^{p}$.

The purpose of this paper is to show that the sums of sparse wavelet expansions can also be characterized by means of atomic decompositions as in (1.4), but made of poorly localized building blocks. More precisely, for all $x_{0} \in \mathbb{R}^{n}$ and $\lambda>0$ we consider the normalized Poisson kernels

$$
P_{\lambda, x_{0}}(x)=\left(1+\left|\lambda\left(x-x_{0}\right)\right|^{2}\right)^{-(n+1) / 2}, \quad x, x_{0} \in \mathbb{R}^{n}, \quad \lambda>0,
$$

and we will show the following:

Theorem 1.1. A tempered distribution $f$ belongs to $\bigcap_{p>0} \dot{B}_{p}^{n / p, p}\left(\mathbb{R}^{n}\right)$ if and only if there exist three sequences $\left(\lambda_{j}\right) \subset \mathbb{R}^{+},\left(x_{j}\right) \subset \mathbb{R}^{n}$ and $\left(c_{j}\right) \subset \mathbb{C}$ such that

$$
\sum_{j=0}^{\infty}\left|c_{j}\right|^{p}<\infty \quad \text { for all } p>0
$$

and

$$
f(x)=\sum_{j=0}^{\infty} c_{j} P_{\lambda_{j}, x_{j}}(x),
$$

the series being uniformly convergent in $\mathbb{R}^{n}$.

In establishing Theorem 1.1 we were motivated by qualitative properties of solutions to evolution equations arising in fluid mechanics (the Euler and the NavierStokes equations). Indeed, consider e.g. the motion of a viscous incompressible fluid filling the whole space $\mathbb{R}^{n}$ and not submitted to the action of external forces. If $u(x, t)$ denotes the velocity of a fluid particle at time $t$, then under suitable assumptions on the vorticity of the flow, it can be shown that $u(\cdot, t) \in \bigcap_{p>0} \dot{B}_{p}^{n / p, p}\left(\mathbb{R}^{n}\right)$, uniformly in time (see [1]). For an application of sparse wavelet expansions to numerical simulations of flows we refer to 15 . 
Here we would like to stress the fact that no physical interpretation can be given to each building block

$$
\alpha(j, k ; t) \psi\left(2^{j} \cdot-k\right)
$$

of the wavelet expansion of $u(\cdot, t)$. In particular, it is not true, as it might be expected, that the motion of the fluid at time $t$ and close to a point of $\mathbb{R}^{n}$, is approximatively given by the terms (1.8) involving small dyadic cubes centred at that point. This is due to the fact that wavelets decay rapidly at infinity, whereas $u$ decays at very slow rates as $x \rightarrow \infty$ : in general not faster than $|x|^{-(n+1)}$, which is the critical decay rate (this is the well-known instantaneous spreading effect for the Navier-Stokes equations; see [2], 6]).

On the other hand, under suitable assumptions, an expansion of the form

$$
u(x, t)=\sum_{m} u_{m}\left(x-x_{m}, t\right),
$$

where $\left(x_{m}\right)$ is a sequence of points in $\mathbb{R}^{n}$ and $u_{m}(\cdot, t)$ are $L^{\infty}$ functions decaying at infinity as $|x|^{-(n+1)}$, holds true (see [1]). Expanding $u(\cdot, t) \in \bigcap_{p>0} \dot{B}_{p}^{n / p, p}\left(\mathbb{R}^{n}\right)$ by means of dilated-translated Poisson kernels (which have the "right" decay rate) is therefore more meaningful, from the physical point of view, than expressing the velocity field as a series of too localized functions, such as gaussians or wavelets.

Poisson kernels being particular rational functions in odd dimension, Theorem 1.1 can also be viewed as a continuation of the work Newmann and Peller on nonlinear approximation. In particular, 13, characterizes the periodic functions of one real variable which can be approximated by rational functions at arbitrarily fast algebraic rates, in the $L^{\infty}$-norm: these functions are exactly those belonging to the Besov spaces $B_{p}^{1 / p, p}$ on the torus, for all $p>0$. Approximation by some specific classes of multivariate rational functions has been recently discussed by Petrushev [14]. In [14, however, the smoothness of functions having a given rate of approximation is not measured by classical Besov spaces but by means of the slightly more involved $B$-spaces. General references for the nonlinear approximation theory are [3] and [4].

The subtle part of the proof of Theorem 1.1 is to show that if $f \in \bigcap_{p>0} \dot{B}_{p}^{n / p, p}\left(\mathbb{R}^{n}\right)$, then (1.7)-(1.6) hold true. Note that, since the decomposition (1.7) is not unique, we cannot deduce the statement by proving our claim for all fixed $p$, since in this case the coefficients $c_{j}$ of (1.7) would depend on $p$. Furthermore, Theorem 1.1 is not a consequence of the general result in 10], since Poisson kernels decay too slowly at infinity. Indeed, the assumptions on the decay of $\Phi$ that we would need to apply [10] become more and more stringent when $p \rightarrow 0$.

Our main tool for establishing our result is a suitable adaptation of an algorithm which was originally developed by Y. Meyer to study the problem of the nonlinear approximation of functions by means of sums of normalized gaussians. This algorithm is described in [11], but since this reference is not widely available, we shall outline its underlining ideas in the next section. In any case, we present the proof of Theorem 1.1 in a such way that our paper will be self-contained, at least for readers with a basic knowledge of Besov spaces.

Notation. For $x=\left(x_{1}, \ldots, x_{n}\right) \in \mathbb{R}^{n}$, we will adopt the following notation: $|x|_{\infty}=$ $\max _{j=1, \ldots, n}\left|x_{j}\right|$ and $|x|=\left(\sum_{j=1}^{n}\left|x_{j}\right|^{2}\right)^{1 / 2}$. 


\section{Proof of Theorem 1.1}

In this paper we use the following definition of the Fourier tranform: if $f \in$ $L^{1}\left(\mathbb{R}^{n}\right)$, we set $\widehat{f}(\xi)=\int f(x) e^{-i x \cdot \xi} d x$. It will be convenient to modify the definition of $P$ in a such way that $\widehat{P}(\xi)=\exp (-|\xi|)$.

Proving that any function of the form (1.7)-(1.6) belongs to $\dot{B}_{p}^{n / p, p}\left(\mathbb{R}^{n}\right)$ for all $p>$ 0 is elementary. Indeed, one first checks with straightforward computations on the wavelet coefficients (1.2) that this is true for $P$. The general result then follows using the invariance of $\dot{B}_{p}^{n / p, p}\left(\mathbb{R}^{n}\right)$ under translation and scaling, and the $p$-triangular inequality

$$
\left\|\sum_{m} f_{m}\right\|_{\dot{B}_{p}^{n / p, p}}^{p} \leq C \sum_{m}\left\|f_{m}\right\|_{\dot{B}_{p}^{n / p, p}}^{p} \quad(0<p \leq 1) .
$$

There is of course no restriction in considering only $0<p \leq 1$, since $\dot{B}_{p}^{n / p, p}\left(\mathbb{R}^{n}\right)$ is embedded in $\dot{B}_{p^{\prime}}^{n / p^{\prime}, p^{\prime}}\left(\mathbb{R}^{n}\right)$ if $0<p \leq p^{\prime}$. The easy part of Theorem 1.1 then follows.

Now let us show that if $f \in \bigcap_{p>0} \dot{B}_{p}^{n / p, p}\left(\mathbb{R}^{n}\right)$, then $f$ can be decomposed as in (1.7)-(1.6). Using again the $p$-triangular inequality and the invariance properties of $\dot{B}_{p}^{n / p, p}\left(\mathbb{R}^{n}\right)$, we see that our claim would immediately follow if we can show that the wavelets $\psi \in F$ can be written in the form (1.7)-(1.6). To do this we shall assume, as we may (see [12]), that $\widehat{\psi}(\xi)$ is a smooth function supported in $\frac{2 \pi}{3} \leq|\xi|_{\infty} \leq \frac{8 \pi}{3}$. Before entering into the details of the proof, we sketch how we are going to obtain such an expansion.

General description of the algorithm. The argument below has been applied in 11] to prove that $\psi$ admits a sparse expansion by means of gaussians, as in (1.4). We present it in a slightly more general form.

Let $g \in L^{1}\left(\mathbb{R}^{n}\right)$ be a function with nonvanishing Fourier transform. The first step consists in writing

$$
\widehat{\psi}(\xi)=\widehat{g}(\xi) p(\xi)-\sum_{\substack{k_{0} \in \mathbb{Z}^{n} \\ k_{0} \neq 0}} R_{k_{0}}(\xi) .
$$

Here we want $p$ to be a periodic function with respect to its variables, with some period $T_{0}$. This is possible if we set $R_{k_{0}}(\xi) \equiv \widehat{\psi}\left(\xi+k_{0} T_{0}\right) \widehat{g}\left(\xi+k_{0} T_{0}\right)^{-1} \widehat{g}(\xi)$. Next each term $R_{k_{0}}$ is treated in a similar way: fix two positive reals $T_{k_{0}}, \beta_{k_{0}}$ and write $R_{k_{0}}(\xi)=\widehat{g}\left(\beta_{k_{0}} \xi\right) p_{k_{0}}(\xi)-\sum_{k_{1} \in \mathbb{Z}^{n}, k_{1} \neq 0} R_{k_{0}, k_{1}}(\xi)$. Here we have defined $R_{k_{0}, k_{1}}(\xi) \equiv R_{k_{0}}\left(\xi+k_{1} T_{k_{0}}\right) \widehat{g}\left(\beta_{k_{0}}\left(\xi+k_{1} T_{k_{0}}\right)\right)^{-1} \widehat{g}\left(\beta_{k_{0}} \xi\right)$, so that $p_{k_{0}}$ is $T_{k_{0}}$-periodic with respect to its variables. After $j$ steps, $\widehat{\psi}$ is written as the sum of terms of the form $\widehat{g}\left(\beta_{k_{0}, \ldots, k_{j}} \xi\right) p_{k_{0}, \ldots, k_{j}}(\xi)$, with $p_{k_{0}, \ldots, k_{j}}$ periodic, plus un error term $E_{j}$. If we forget for a moment the error, then taking $j \rightarrow \infty$ and the inverse Fourier transform we see that, at least formally, $\psi$ is written as a series of dilated and shifted functions of the function $g$.

Several nice properties of the Poisson kernel allow us to make this argument rigorous, when $g=P$. Of course, the scale factors $\beta_{k_{0}, \ldots, k_{j}}$ and the periods $T_{k_{0}, \ldots, k_{j}}$ must be chosen in a suitable way. Condition (1.6) will follow from some careful estimates on the size of the Fourier coefficients of the periodic functions involved.

To avoid the proliferation of too many subscripts, from now on we will write $\mathbf{k}(0), \mathbf{k}(1) \ldots$, instead of $k_{0}, k_{1}, \ldots$ 
Application to the Poisson kernel: the first step. We start with the defininition of a tree $\Lambda$. The $j$-th generation of $\Lambda(j=0,1, \ldots)$ is the set $\Lambda_{j}$ of $n \times(j+1)$ matrices such that

$$
\Lambda_{j}=\left\{\lambda(j)=(\mathbf{k}(0), \ldots, \mathbf{k}(j)) \mid \mathbf{k}(0), \ldots, \mathbf{k}(j) \in \mathbb{Z}^{n}, \mathbf{k}(0) \neq \mathbf{0}, \ldots, \mathbf{k}(j) \neq \mathbf{0}\right\},
$$

where $\mathbf{0}=(0, \ldots, 0) \in \mathbb{R}^{n}$. Then we set

$$
\Lambda=\{\mathbf{0}\} \cup \bigcup_{j=0}^{\infty} \Lambda_{j} .
$$

A matrix $\lambda^{\prime} \in \Lambda_{j+1}$ is a son of $\lambda \in \Lambda_{j}$ if $\lambda^{\prime}$ is obtained from $\lambda$ by adding the last column, i.e. if $\lambda^{\prime}=(\lambda, \mathbf{k}(j+1))$ for some vector $\mathbf{k}(j+1) \in \mathbb{Z}^{n}$.

For all $j \geq 0$ and all $\lambda=\lambda(j) \in \Lambda_{j}$ let us define $\xi_{\lambda} \in \mathbb{R}^{n}, \beta_{\lambda}>0$ and $T_{\lambda}>0$ in the following way:

$$
\begin{array}{ll}
\xi_{\mathbf{0}}=0, \quad \beta_{\mathbf{0}}=1, & T_{\mathbf{0}}=16 \pi / 3, \\
\xi_{\lambda(0)}=\mathbf{k}(0) T_{\mathbf{0}}, & T_{\lambda}=(j+2)\left|\xi_{\lambda}\right|, \\
\xi_{\lambda^{\prime}}=\xi_{\lambda}+\mathbf{k}(j+1) T_{\lambda} & \beta_{\lambda}=\left|\xi_{\lambda}\right|^{-1}
\end{array}
$$

(we might choose $T_{\mathbf{0}}$ arbitrarily large).

Next we define three families of functions, $f_{\lambda}, g_{\lambda}$ and $p_{\lambda}$, indexed on the tree $\Lambda$ :

$$
\begin{aligned}
& f_{\mathbf{0}}(\xi)=\widehat{\psi}(\xi) \\
& f_{\lambda(0)}(\xi)=\exp (-|\xi|) \exp \left(\left|\xi-\mathbf{k}(0) T_{\mathbf{0}}\right|\right) f_{\mathbf{0}}\left(\xi-\mathbf{k}(0) T_{\mathbf{0}}\right), \\
& f_{\lambda^{\prime}}(\xi)=\exp \left(-\beta_{\lambda}|\xi|\right) g_{\lambda}\left(\xi-\mathbf{k}(j+1) T_{\lambda}\right) \\
& g_{\lambda}(\xi)=\exp \left(\beta_{\lambda}|\xi|\right) f_{\lambda}(\xi) \\
& p_{\lambda}(\xi)=\sum_{\mathbf{k} \in \mathbb{Z}^{n}} g_{\lambda}\left(\xi-\mathbf{k} T_{\lambda}\right) .
\end{aligned}
$$

Note that the convergence of the series defining $p_{\lambda}$ is ensured by the fact that both $f_{\lambda}$ and $g_{\lambda}$ are compactly supported for all $\lambda \in \Lambda$. On the other hand, $p_{\lambda}$ is $T_{\lambda}$-periodic with respect to all its variables, hence

$$
p_{\lambda}(\xi)=\sum_{m \in \mathbb{Z}^{n}} \alpha_{\lambda}(m) \exp \left(i \frac{2 \pi}{T_{\lambda}} m \cdot \xi\right)
$$

(we will see in Lemma 2.3 below that the Fourier series (2.3) is absolutely convergent), where

$$
\alpha_{\lambda}(m)=\frac{1}{T_{\lambda}^{n}} \int_{0}^{T_{\lambda}} \cdots \int_{0}^{T_{\lambda}} p_{\lambda}(\xi) \exp \left(-i \frac{2 \pi}{T_{\lambda}} m \cdot \xi\right) d \xi .
$$

Simple substitutions in 2.2. show that we can "climb the tree" and determine $f_{\lambda}$ if we know $f_{\lambda^{\prime}}$ for all sons $\lambda^{\prime}$ of $\lambda$ :

$$
f_{\lambda}(\xi)=\exp \left(-\beta_{\lambda}|\xi|\right) p_{\lambda}(\xi)-\sum_{\left\{\lambda^{\prime} \text { son of } \lambda\right\}} f_{\lambda^{\prime}}(\xi) .
$$

Hence, for all $J \in \mathbb{N}$,

$$
f_{\mathbf{0}}(\xi)=\exp (-|\xi|) p_{\mathbf{0}}(\xi)-\sum_{j=0}^{J}(-)^{j} \sum_{\lambda \in \Lambda_{j}} \exp \left(-\beta_{\lambda}|\xi|\right) p_{\lambda}(\xi)+(-)^{J} \sum_{\lambda \in \Lambda_{J}} f_{\lambda}(\xi)
$$


Here $\sum_{\lambda \in \Lambda_{J}} f_{\lambda}(\xi)$ is an error term. We claim (see Remark 2.2 below for a proof) that this term converges uniformly to 0 as $J \rightarrow \infty$. It then follows that

$$
\widehat{\psi}(\xi)=\exp (-|\xi|) p_{\mathbf{0}}(\xi)-\sum_{j=0}^{\infty}(-)^{j} \sum_{\lambda \in \Lambda_{j}} \exp \left(-\beta_{\lambda}|\xi|\right) p_{\lambda}(\xi) .
$$

Taking the inverse Fourier transform, we then see that $\psi(x)$ is the sum of a series, indexed over the tree $\Lambda$, of terms of the form (up to an unessential sign)

$$
\sum_{m \in \mathbb{Z}^{n}} \alpha_{\lambda}(m) \beta_{\lambda}^{-n} P\left(\frac{x+2 \pi T_{\lambda}^{-1} m}{\beta_{\lambda}}\right) .
$$

Collecting all the terms (2.8) yields the atomic decomposition (1.7). Then the proof will be finished if we show that

$$
\sum_{\lambda \in \Lambda} \sum_{m \in \mathbb{Z}^{n}}\left|\alpha_{\lambda}(m)\right|^{p} \beta_{\lambda}^{-n p}<\infty, \quad \text { for all } p>0 .
$$

The second step. In the second part of the proof we will use several nice properties of $\widehat{P}(\xi)$.

Lemma 2.1. Let $\lambda=\lambda(j)$ in $\Lambda_{j}$. Then we have

$$
f_{\lambda}(\xi)=\exp \left(a_{\lambda}\left(\xi-\xi_{\lambda}\right)-b_{\lambda}\right) f_{\mathbf{0}}\left(\xi-\xi_{\lambda}\right), \quad \xi \in \mathbb{R}^{n},
$$

where

$$
b_{\lambda}=\frac{\left|\xi_{\lambda(j)}\right|}{\left|\xi_{\lambda(j-1)}\right|}+\cdots+\frac{\left|\xi_{\lambda(1)}\right|}{\left|\xi_{\lambda(0)}\right|}+\left|\xi_{\lambda(0)}\right|-j
$$

and $a_{\lambda}$ are $C^{\infty}$ functions on the support of $f_{0}=\widehat{\psi}$, such that for all multi-index $\alpha \in \mathbb{N}^{n}$ there exists a constant $C_{\alpha}$ satisfying

$$
\left|\partial_{\xi}^{\alpha} a_{\lambda}(\xi)\right| \leq C_{\alpha}
$$

uniformly in $\lambda$ and $\xi$ (for $\frac{2 \pi}{3} \leq|\xi|_{\infty} \leq \frac{8 \pi}{3}$ ).

Proof. The case $j=0$ is obvious since we already observed in (2.2) that

$$
f_{\lambda(0)}(\xi)=\exp \left(-|\xi|+\left|\xi-\mathbf{k}(0) T_{\mathbf{0}}\right|\right) f_{\mathbf{0}}\left(\xi-\xi_{\lambda(0)}\right) .
$$

Then it is sufficient to take

$$
\begin{aligned}
& b_{\lambda(0)}=|\mathbf{k}(0)| T_{\mathbf{0}}=\left|\xi_{\lambda(0)}\right|, \\
& a_{\lambda(0)}(\xi)=|\xi|-\left|\xi+\xi_{\lambda(0)}\right|+\left|\xi_{\lambda(0)}\right|
\end{aligned}
$$

to see that $f_{\lambda(0)}$ can be written as in (2.10). It follows from (2.2), (2.10) and $\xi_{\lambda^{\prime}}=\xi_{\lambda}+\mathbf{k}(j+1) T_{\lambda}$ that

$$
\begin{aligned}
f_{\lambda^{\prime}}(\xi) & =\exp \left(a_{\lambda}\left(\xi-\xi_{\lambda^{\prime}}\right)-\beta_{\lambda}|\xi|+\beta_{\lambda}\left|\xi-\mathbf{k}(j+1) T_{\lambda}\right|-b_{\lambda}\right) f_{\mathbf{0}}\left(\xi-\xi_{\lambda^{\prime}}\right) \\
& =\exp \left(a_{\lambda}\left(\xi-\xi_{\lambda^{\prime}}\right)+\beta_{\lambda} h_{\lambda^{\prime}}\left(\xi-\xi_{\lambda^{\prime}}\right)-b_{\lambda^{\prime}}\right) f_{\mathbf{0}}\left(\xi-\xi_{\lambda^{\prime}}\right),
\end{aligned}
$$

where

$$
\begin{aligned}
& b_{\lambda^{\prime}}=b_{\lambda}+\beta_{\lambda}\left|\xi_{\lambda^{\prime}}\right|-\beta_{\lambda}\left|\xi_{\lambda}\right|=b_{\lambda}+\left|\xi_{\lambda^{\prime}}\right| /\left|\xi_{\lambda}\right|-1, \\
& h_{\lambda^{\prime}}(\xi)=-\left|\xi+\xi_{\lambda^{\prime}}\right|+\left|\xi+\xi_{\lambda}\right|+\left|\xi_{\lambda^{\prime}}\right|-\left|\xi_{\lambda}\right| .
\end{aligned}
$$

This yields (2.11). Next we set

$$
a_{\lambda^{\prime}}(\xi)=a_{\lambda}(\xi)+\beta_{\lambda} h_{\lambda^{\prime}}(\xi) .
$$


Since the derivatives of $h_{\lambda^{\prime}}$ are uniformly bounded on the support of $f_{\mathbf{0}}$ and $\beta_{\lambda} \leq$ $1 / j$ !, we get (2.10) and (2.12).

Remark 2.2. It follows from (2.1) and this lemma that whenever $\lambda$ and $\tilde{\lambda}$ belong to $\Lambda$ and $\lambda \neq \tilde{\lambda}$, then $f_{\lambda}$ and $f_{\tilde{\lambda}}$ have disjoint supports. Thus, if we come back to the error-terms contained in (2.6), we see that

$$
\left\|\sum_{\lambda \in \Lambda_{J}} f_{\lambda}\right\|_{\infty} \leq C \sup _{\lambda \in \Lambda_{J}} \exp \left(-b_{\lambda}\right) \rightarrow 0, \quad \text { as } J \rightarrow \infty .
$$

Let us now establish (2.9). The proof is based on the following elementary lemma.

Lemma 2.3. Let $0<p \leq 1, N>n / p$ and $g \in L^{1}\left(\mathbb{R}^{n}\right)$ such that

$$
|\widehat{g}(\eta)| \leq A(1+|\eta|)^{-N} \quad \text { for all } \eta \in \mathbb{R}^{n} .
$$

For all $T>0$, the function $p(\xi)=\sum_{\mathbf{k} \in \mathbb{Z}^{n}} g(\xi-\mathbf{k} T)$ is T-periodic with respect to its variables and if $\sum_{m \in \mathbb{Z}^{n}} \alpha(m) \exp \left(i \frac{2 \pi}{T} m \cdot x\right)$ denotes its Fourier series, then we have

$$
\sum_{m \in \mathbb{Z}^{n}}|\alpha(m)|^{p} \leq C A^{p} T^{n(1-p)} .
$$

Proof. The Fourier coefficients of $p$ are $\alpha(m)=T^{-n} \widehat{g}\left(\frac{2 \pi}{T} m\right)$, hence

$$
\sum_{m \in \mathbb{Z}^{n}}|\alpha(m)|^{p} \leq\left(\sum_{|m| \leq T / 2 \pi}+\sum_{|m|>T / 2 \pi}\right) \frac{A^{p} T^{-n p}}{\left(1+\frac{2 \pi}{T}|m|\right)^{N p}}
$$

and the conclusion of Lemma 2.3 is now immediate.

We now apply this lemma to

$$
\begin{aligned}
g_{\lambda}(\xi) & =\exp \left(\beta_{\lambda}|\xi|\right) f_{\lambda}(\xi) \\
& =\exp \left(a_{\lambda}\left(\xi-\xi_{\lambda}\right)\right) \exp \left(\beta_{\lambda}|\xi|\right) \exp \left(-b_{\lambda}\right) f_{\mathbf{0}}\left(\xi-\xi_{\lambda}\right) .
\end{aligned}
$$

From now on $p$ and the integer $N$ will be fixed $(0<p \leq 1, N>n / p)$ and in our estimates we will denote by $C$ a constant, which may change from line to line, but depends only on $p, N$ and $n$.

We start by observing that $g_{\lambda} \in C_{0}^{\infty}\left(\mathbb{R}^{n}\right)$ and that

$$
|\eta|^{N}\left|\widehat{g}_{\lambda}(\eta)\right| \leq \int_{2 \pi / 3 \leq\left|\xi-\xi_{\lambda}\right| \leq 8 \pi / 3}\left|\partial_{\xi}^{N} g_{\lambda}(\xi)\right| d \xi .
$$

This integral is bounded by $C \exp \left(-b_{\lambda}\right)$. This follows from the fact that the derivatives of $\exp \left(a_{\lambda}\left(\xi-\xi_{\lambda}\right)\right) \exp \left(\beta_{\lambda}|\xi|\right)$ are uniformly bounded with respect to $\lambda$ and $\xi$, on the set of the $\xi$ such that $\frac{2 \pi}{3} \leq\left|\xi-\xi_{\lambda}\right|_{\infty} \leq \frac{8 \pi}{3}$ (we use here that $\beta_{\lambda} \rightarrow 0$ ). From Lemma 2.3 we see that Fourier coefficients $\alpha(m)$, defined by (2.3) and (2.4), satisfy

$$
\sum_{m \in \mathbb{Z}^{n}}\left|\alpha_{\lambda}(m)\right|^{p} \leq C T_{\lambda}^{n(1-p)} \exp \left(-p b_{\lambda}\right) .
$$

The proof of (2.9) and of Theorem 1.1 then will be finished if we are able to prove that the series

$$
\sum_{j=0}^{\infty} \sum_{\lambda \in \Lambda_{j}} j^{n}\left|\xi_{\lambda(j)}\right|^{n} \exp \left(-p b_{\lambda(j)}\right)
$$


converges. The $j=0$ term of this series can be treated in an obvious way, so we can just consider $j \geq 1$. The bound of $\left|\xi_{\lambda(j)}\right|^{n} \exp \left(-p b_{\lambda(j)}\right)$ relies on (2.11) and the elementary inequality $y \exp (-p y) \leq C \exp (-p y / 2)$. This gives us

$$
\begin{aligned}
\left|\xi_{\lambda(j)}\right|^{n} \exp \left(-p b_{\lambda(j)}\right) \leq & C^{j+1} \exp \left(-\frac{p\left|\xi_{\lambda(j)}\right|}{2\left|\xi_{\lambda(j-1)}\right|}\right) \\
& \times \cdots \times \exp \left(-\frac{p\left|\xi_{\lambda(1)}\right|}{2\left|\xi_{\lambda(0)}\right|}\right) \exp \left(-\frac{p\left|\xi_{\lambda(0)}\right|}{2}\right) \exp (p j) .
\end{aligned}
$$

But $\lambda(j)=(\mathbf{k}(0), \ldots, \mathbf{k}(j))$ and $\frac{\left|\xi_{\lambda(l)}\right|}{\left|\xi_{\lambda(l-1)}\right|} \geq l|\mathbf{k}(l)|$ for $l=1, \ldots, j$. Hence,

$$
\begin{aligned}
\sum_{\lambda \in \Lambda_{j}}\left|\xi_{\lambda(j)}\right|^{n} & \exp \left(-p b_{\lambda(j)}\right) \\
& \leq C^{j} \exp (p j) \sum_{\mathbf{k}(0) \neq \mathbf{0}} \ldots \sum_{\mathbf{k}(j) \neq \mathbf{0}} \exp \left(-\frac{p j|\mathbf{k}(j)|}{2}\right) \times \cdots \times \exp \left(-\frac{p|\mathbf{k}(0)|}{2}\right) \\
& \leq C^{j} \exp (-p j / 4) \exp (-p(j-1) / 4) \times \cdots \times \exp (-p / 4) \\
& \leq C^{j} \exp \left(-p j^{2} / 8\right)
\end{aligned}
$$

and Theorem 1.1 follows.

\section{ACKNOWLEDGEMENTS}

The discussions with Yves Meyer on the problem treated in this paper are gratefully acknowledged.

\section{REFERENCES}

[1] L. Brandolese, Atomic decomposition for the vorticity of a viscous flow in the whole space, Math. Nachr. 273, 28-42 (2004). MR2084955

[2] L. Brandolese, Y. Meyer, On the instantaneous spreading for the Navier-Stokes system in the whole space, ESAIM Contr. Optim. Calc. Var. 8, 273-285 (2002). MR 1932953 (2003j:35246)

[3] R. DeVore, Nonlinear approximation, Acta Numer. 1-99 (1998). MR1689432 (2001a:41034)

[4] R. DeVore, B. Jawerth, V. Popov, Compression of wavelet decompositions, Amer. J. of Math. 114, 737-785 (1992). MR:1175690(94a:42045)

[5] D. Donoho, I. M. Johnstone, G. Kerkiacharian, D. Picard, Wavelet shrinkage: Asymptopia? J. Roy. Statist. Soc. Ser B 57, 301-369 (1995). MR1323344 (96g:62068)

[6] S. Y. Dobrokhotov, A. I. Shafarevich, Some Integral Identities and Remarks on the Decay at Infinity of the Solutions to the Navier-Stokes Equations in the Entire Space, Russ. J. Math. Phys. Vol. 2, No. 1, 133-135 (1994). MR:1297948 (95f:35194)

[7] M. Frazier, B. Jawerth, G. Weiss, The Littlewood-Paley Theory and the study of Function spaces, CBMS Regional Conf. Series in Math., 79, Amer. Math. Soc., Providence (1991). MR $1107300(92 \mathrm{~m}: 42021)$

[8] S. Jaffard, Y. Meyer, On the pointwise regularity of functions in critical Besov Spaces, J. of Funct. Anal. 175, 415-434 (2000). MR,1780484 (2001f:46047)

[9] S. Jaffard, Y. Meyer, R. Ryan, Wavelets, tools for science $\&$ technology. Revised edition, Society for Industrial and Applied Mathematics (SIAM), Philadelphia, PA 2001. MR 1827998 (2002g:00007)

[10] G. Kyriazis, P. Petrushev, New bases for Tribel-lizorkin and Besov Spaces, Trans. Amer. Math. Soc. 354, No. 2, 749-786 (2002). MF1862566 (2002k:46082)

[11] Y. Meyer, Un problème mathématique lié à la compression (manuscript).

[12] Y. Meyer, Ondelettes et opérateurs, Vol. 1, Hermann, Paris (1990). MR.1085487 (93i:42002)

[13] V. Peller, Henkel operators of the class $S_{p}$, investigations on the rate of rational approximations and other applications, Mat. Sb. 122, 481-510 (1980). MR0725454 (85g:47041) 
[14] P. Petrushev, Multivariate -term rational and piecewise polynomial approximation, J. Approx. Theory 121, No. 1, 128-197 (2003). MR1963001 (2003m:41054)

[15] K. Schneider, M. Farge, Numerical simulation of a mixing layer in an adaptive wavelet basis, C. R. Acad. Sci. Paris t. 328, Série II, b, Mécanique des fluides, 263-269 (2000).

Institut Camille Jordan, Université Lyon 1, 21 avenue Claude Bernard, 69622 Villeurbanne Cedex, France

E-mail address: brandolese@igd.univ-lyon1.fr 